\title{
Reduced-Complexity Iterative Markov Chain MBER Detection for MIMO Systems
}

\author{
Shinya Sugiura, Member, IEEE, Sheng Chen, Senior Member, IEEE, and Lajos Hanzo, Fellow, IEEE
}

\begin{abstract}
A novel Markov chain (MC) representation aided minimum bit error rate (MBER) detection method is proposed for an $\mathcal{M}$-QAM modulated SDM/SDMA uplink system. Compared to the conventional MBER scheme, the proposed MC-MBER scheme is capable of reducing the complexity imposed with the aid of its efficient detection candidate set generation assisted by the Markov chain process. Our performance results demonstrate that the MC-MBER multiuser detection (MUD) is capable of reducing the computational complexity by a factor of eight in comparison to the conventional MBER MUD in a rank-deficient system transmitting four 4-QAM substreams with the aid of two receive antennas, while achieving a BER performance comparable to that of the MBER MUD.
\end{abstract}

Index Terms-Markov chain Monte Carlo, minimum bit error rate, multiuser detection, soft interference cancellation.

\section{INTRODUCTION}

$\mathbf{S}$ INCE the invention of turbo codes by Berrou et al. [1], iterative linear detection based on the minimum mean square error (MMSE) criterion and exploiting the a priori information gleaned from a second decoder component has been investigated in diverse receivers [2], [3]. Although the MMSE detection criterion has been widely used for iterative multiuser detections (MUDs), minimizing the MSE does not necessarily guarantee the direct minimization of the system's bit error ratio (BER). By contrast, the family of minimum BER (MBER) detectors [4]-[7] was designed to directly minimize the BER, and hence, it was shown to outperform the MMSE solution. However, the high BER performance of the MBER scheme is achieved at the cost of a high computational complexity, which may become particularly challenging in rapidly fading propagation environments, requiring prompt MBER detector weight updates or in iterative detection scenarios, where soft information has to be exchanged between the detector and the channel decoder. In recent years, studies of Markov chain simulations have found reduced-complexity applications in wireless communication systems [8]-[11]. More specifically in [11], the iterative nonlinear Markov chain Monte Carlo (MCMC)-based detector was designed with the aid of multidimensional Monte Carlo integration for low-complexity near-optimum MUD.

Manuscript received August 09, 2008; revised October 30, 2008. Current version published February 11, 2009. The associate editor coordinating the review of this manuscript and approving it for publication was Dr. Robert Fischer.

S. Sugiura is with the School of Electronics and Computer Science, University of Southampton, Southampton SO17 1BJ, U.K., and also with the Toyota Central Research and Development Laboratories, Inc., Aichi 480-1192, Japan (e-mail: ss07r@ecs.soton.ac.uk).

C. Chen and L. Hanzo are with the School of Electronics and Computer Science, University of Southampton, Southampton SO17 1BJ, U.K. (e-mail: sqc@ecs.soton.ac.uk; lh@ecs.soton.ac.uk).

Digital Object Identifier 10.1109/LSP.2008.2010817

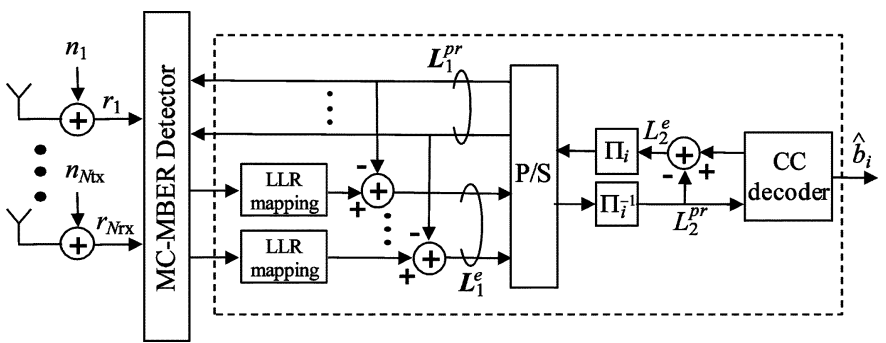

Fig. 1. Iterative receiver structure of an SDM/SDMA uplink.

Against this background, the novel contribution of this letter is that a Markov chain representation aided MBER algorithm is proposed for the sake of reducing the computational complexity of the conventional MBER algorithm without degrading its performance.

\section{SySTEM OVERVIEW}

\section{A. System Description}

Assume an SDM/SDMA uplink scenario, where each of the $N_{u}$ users has $N_{\text {tx }}$ transmit antennas, while the base station (BS) is equipped with an $N_{\mathrm{rx}}$-element antenna array. At the $i$ th user, the source bits $b_{i}$ are first channel encoded and then interleaved by the user-specific interleaver $\Pi_{i}$. Next, the interleaved bits are $\mathrm{S} / \mathrm{P}$ converted to $N_{\mathrm{tx}}$ substreams and then mapped to the $\mathcal{M}$-QAM symbols $\boldsymbol{s}_{i}=\left[s_{i}^{(1)}, \ldots, s_{i}^{\left(N_{\mathrm{tx}}\right)}\right]^{T}$. Finally, a total of $N_{u} N_{\mathrm{tx}} \mathcal{M}$-QAM symbols are simultaneously transmitted via each transmit antenna of each user. For simplicity, we consider perfectly synchronous transmissions of all the users, which would require accurate adaptive timing advance control.

The receiver structure is shown in Fig. 1. Based on the turbo detection principle, the receiver employs an iterative MUD in the SDM/SDMA uplink. The receiver consists of two soft-input soft-output (SISO) stages, namely, the SISO interference cancellation aided MUD and $N_{u}$ number of parallel single-user SISO channel decoders. The MUD scheme employed demodulates the received symbols $\boldsymbol{r}$ and outputs the extrinsic information $\boldsymbol{L}_{1}^{e}$ in the form of $\log$ likelihood ratios (LLRs) with the aid of the a priori LLRs $\boldsymbol{L}_{1}^{p r}$, which are fed back from the channel decoders to the detector. The extrinsic LLRs $\boldsymbol{L}_{1}^{e}$ are then input to the convolutional channel (CC) decoders of Fig. 1 after deinterleaving. The extrinsic LLRs $L_{2}^{e}$ of Fig. 1 are calculated at the channel decoders, output and are interleaved again, before being passed back to the MUD component as the a priori information.

\section{B. Signal Model}

Under the assumption of a frequency-flat channel environment, the received signals $\boldsymbol{r} \in \mathcal{C}^{N_{\mathrm{rx}} \times 1}$ are given by 
the complex-valued expression of $\boldsymbol{r}=\boldsymbol{H} \boldsymbol{s}+\boldsymbol{n}$, where $\boldsymbol{s}=\left[\boldsymbol{s}_{1}^{T} \cdots \boldsymbol{s}_{N_{u}}^{T}\right]^{T} \in \mathcal{C}^{N_{u} N_{\mathrm{tx}} \times 1}$ are the $\mathcal{M}$-QAM symbols and $\boldsymbol{n} \in \mathcal{C}^{N_{\mathrm{rx}} \times 1}$ are the corresponding noise components having a zero mean and a power of $2 \sigma_{n}^{2}$. Furthermore, $\boldsymbol{H} \in \mathcal{C}^{N_{\mathrm{rx}} \times N_{\mathrm{tx}}}$ denotes the channel matrix.

Considering the MBER and MC-MBER schemes, it is convenient to represent the estimated signals by real-valued binary expressions. Let us hence consider the transformation of the above complex-valued $\mathcal{M}$-QAM signal model to the equivalent real-valued binary signal model.

Let us assume first of all that the $i$ th user's and the $l$ th antenna's $\mathcal{M}$-QAM symbol $s_{i}^{(l)}$ as well as the equivalent realvalued binary symbols $\tilde{\boldsymbol{b}}_{i}^{(l)} \in \mathcal{R}^{\log _{2}(\mathcal{M}) \times 1}$ have the relationship of $s_{i}^{(l)}=\boldsymbol{q} \tilde{\boldsymbol{b}}_{i}^{(l)}$, where

$$
\boldsymbol{q}= \begin{cases}{\left[\frac{1}{\sqrt{2}}-\frac{j}{\sqrt{2}}\right]} & (\mathcal{M}=4) \\ {\left[\frac{2}{\sqrt{10}} \frac{1}{\sqrt{10}}-\frac{2 j}{\sqrt{10}}-\frac{j}{\sqrt{10}}\right]} & (\mathcal{M}=16) .\end{cases}
$$

Then, the equivalent real-valued binary signal model is given by

$$
\boldsymbol{y}=\boldsymbol{H}^{\prime} \boldsymbol{x}+\boldsymbol{\eta}
$$

where we have

$$
\begin{aligned}
\boldsymbol{y} & =\left[\Re(\boldsymbol{r})^{T} \Im(\boldsymbol{r})^{T}\right]^{T} \in \mathcal{R}^{N \times 1} \\
\boldsymbol{\eta} & =\left[\Re(\boldsymbol{n})^{T} \Im(\boldsymbol{n})^{T}\right]^{T} \in \mathcal{R}^{N \times 1} \\
\boldsymbol{x} & =\left[\overline{\boldsymbol{b}}_{1}^{T} \cdots \overline{\boldsymbol{b}}_{N_{u}}^{T}\right]^{T} \in \mathcal{R}^{M \times 1} \\
\boldsymbol{H}^{\prime} & =\left[\Re(\boldsymbol{H} \boldsymbol{Q})^{T} \Im(\boldsymbol{H} \boldsymbol{Q})^{T}\right]^{T} \in \mathcal{R}^{N \times M}
\end{aligned}
$$

with $M=N_{u} N_{\text {tx }} \log _{2}(\mathcal{M}), N=2 N_{\mathrm{rx}}$, $\overline{\boldsymbol{b}}_{i}=\left[\tilde{\boldsymbol{b}}_{i}^{(1) T} \cdots \tilde{\boldsymbol{b}}_{i}^{\left(N_{\mathrm{tx}}\right) T}\right]^{T}$, and $\boldsymbol{Q}=\boldsymbol{I}_{M} \otimes \boldsymbol{q}$. Note that the equivalent real-valued noise components $\boldsymbol{\eta}$ have the power of $\sigma_{n}^{\prime 2}=\sigma_{n}^{2}$. Throughout the rest of this letter, we employ this real-valued signal model.

\section{MARKov Chain MBER Detection}

In this section, we present our novel detection algorithm, following a brief introduction of the conventional MBER scheme.

\section{A. Conventional MBER Detection}

Let us define the $N_{b}=2^{M}$ number of legitimate transmitted sequences of $\boldsymbol{x}$ as $\boldsymbol{x}^{(q)} \quad\left(q=1, \ldots N_{b}\right)$, with $M=$ $N_{u} N_{\mathrm{tx}} \log _{2}(\mathcal{M})$. Then, the error probability of the $m$ th substream signal $x_{m}$ can be expressed as [6]

$$
P_{e}\left(\boldsymbol{w}_{m}\right)=\sum_{q=1}^{N_{b}} P\left(\boldsymbol{x}^{(q)}\right) \cdot Q\left[\frac{\operatorname{sgn}\left(x_{m}^{(q)}\right) \cdot \breve{x}_{m}^{(q)}}{\sigma_{n}^{\prime} \sqrt{\boldsymbol{w}_{m}^{T} \boldsymbol{w}_{m}}}\right]
$$

with $\check{x}_{m}^{(q)}=\boldsymbol{w}_{m}^{T}\left(\boldsymbol{H}^{\prime} \boldsymbol{x}^{(q)}-\boldsymbol{H}^{\prime} \overline{\boldsymbol{x}}+\bar{x}_{m} \boldsymbol{h}_{m}^{\prime}\right)$, where $P\left(\boldsymbol{x}^{(q)}\right)=\Pi_{m} P\left(x_{m}=x_{m}^{(q)}\right)$ is the a priori probability of transmitting $\boldsymbol{x}^{(q)}$, and $Q[]$ is the Gaussian Q-function. Furthermore, $\boldsymbol{w}_{m} \in \mathcal{R}^{N \times 1}$ is the weight vector corresponding to $x_{m}$ and $\boldsymbol{h}_{m}^{\prime}$ is the $m$ th column of $\boldsymbol{H}^{\prime}$. The soft estimates $\overline{\boldsymbol{x}}=\left[\bar{x}_{1} \cdots \bar{x}_{M}\right]^{T}$ are computed from the a priori LLRs $\boldsymbol{L}_{1}^{p r}=\left[L_{1, x_{1}}^{p r} \cdots L_{1, x_{M}}^{p r}\right]^{T}$ as $\bar{x}_{m}=\tanh \left(L_{1, x_{m}}^{p r} / 2\right)$. The MBER weights are derived by minimizing the BER function of (6) as follows:

$$
\boldsymbol{w}_{m, m b e r}=\arg \min _{\boldsymbol{w}} P_{e}\left(\boldsymbol{w}_{m}\right)
$$

In (6), the probability $P_{e}\left(\boldsymbol{w}_{m}\right)$ is a nonlinear function of the weights $\boldsymbol{w}_{i}$; therefore, in general, the optimization problem has to be solved iteratively. Since the gradient of (6) is given by [6]

$$
\begin{aligned}
& \nabla P_{e}\left(\overline{\boldsymbol{w}}_{m}\right)=\frac{1}{\sqrt{2 \pi} \sigma_{n}^{\prime}} \sum_{q=1}^{N_{b}} P\left(\boldsymbol{x}^{(q)}\right) \exp \left(-\frac{\left(\check{x}_{m}\right)^{2}}{2 \sigma_{n}^{\prime 2}}\right) \\
& \cdot \operatorname{sgn}\left(x_{m}^{(q)}\right) \cdot\left(\overline{\boldsymbol{w}}_{m} \check{x}_{m}^{(q)}-\left(\boldsymbol{H}^{\prime} \boldsymbol{x}^{(q)}-\boldsymbol{H}^{\prime} \overline{\boldsymbol{x}}^{(q)} \bar{x}_{m} \boldsymbol{h}_{m}^{\prime}\right)\right)
\end{aligned}
$$

with $\overline{\boldsymbol{w}}_{m}=\boldsymbol{w}_{m} / \sqrt{\boldsymbol{w}_{m}^{T} \boldsymbol{w}_{m}}$, the simplified conjugate gradient (SCG) algorithm [4] provides an efficient solution for this optimization problem. As described in [6], the symbols estimated by the MBER detector is non-Gaussian. Thus, the exact expression of the extrinsic information has to be employed, which is given by

$$
L_{1, x_{m}}^{e}=\ln \frac{\sum_{x_{m}^{(q)}=0} P\left(\hat{x}_{m} \mid \boldsymbol{x}^{(q)}\right) \Pi_{m^{\prime} \neq m} P\left(x_{m^{\prime}}^{(q)}\right)}{\sum_{x_{m}^{(q)}=1} P\left(\hat{x}_{m} \mid \boldsymbol{x}^{(q)}\right) \Pi_{m^{\prime} \neq m} P\left(x_{m^{\prime}}^{(q)}\right)}
$$

where we have

$$
\begin{aligned}
P\left(\hat{x}_{m} \mid \boldsymbol{x}^{(q)}\right)= & \frac{1}{\sqrt{2 \pi} \sigma_{n}^{\prime}} \\
& \times \exp \left(-\frac{\left[\overline{\boldsymbol{w}}_{m}^{T}\left(\boldsymbol{y}-\boldsymbol{H}^{\prime} \boldsymbol{x}^{(q)}\right)\right]^{2}}{2 \sigma_{n}^{\prime 2}}\right) \\
P\left(x_{m^{\prime}}^{(q)}\right)= & \frac{\left[1+\operatorname{sgn}\left(x_{m^{\prime}}^{(q)}\right) \tanh \left(\frac{L_{1, x_{m}}^{p r}}{2}\right)\right]}{2} .
\end{aligned}
$$

Clearly, the calculation of the MBER weight gradient in (8) imposes a high computational complexity, which increases exponentially with the value of $M$. It may be readily shown that an unlikely signal set of $\boldsymbol{x}^{(q)}$ resulting in a small value of $P\left(\boldsymbol{x}^{(q)}\right)$ does not substantially contribute to the gradient expression of (8). Thus, we introduce the Markov chain (MC) representation method that efficiently extracts a likely set of signals from the $N_{b}=2^{M}$ legitimate sequences for the sake of reducing the computational complexity associated with the gradient calculation in (8) without degrading the BER performance of the full-complexity MBER scheme.

\section{B. Principle of Markov Chain MBER Detection}

The MCMC algorithm is based on two different techniques, i.e., MC representation and Monte Carlo integration. While the former is employed to find the most likely detection candidates according to the associated probability distributions, the latter is used to approximate the integral of interest on the basis of the detection candidates calculated by the $\mathrm{MC}$ representation. In our MC-MBER detector, only the MC representation is used to generate the most likely $N_{b}^{\prime}<N_{b}$ number of signals $\boldsymbol{x}^{\left(q^{\prime}\right)}\left(q^{\prime}=\right.$ $\left.1, \ldots, N_{b}^{\prime}\right)$, which are our detection candidates in this letter. The detection candidates are then input to the MBER detector.

Several algorithms have been designed for finding the most likely decision candidate set with the aid of an MC process [10]. In this contribution, we employ the most popular so-called Gibbs-Sampler, which assists us in sampling the detection candidates set, with the aim of finding the most likely ones [11]. Fig. 2 portrays a flowchart of the Gibbs-Sampler algorithm employed in our SDM/SDMA system, where the algorithmic steps are as follows. 


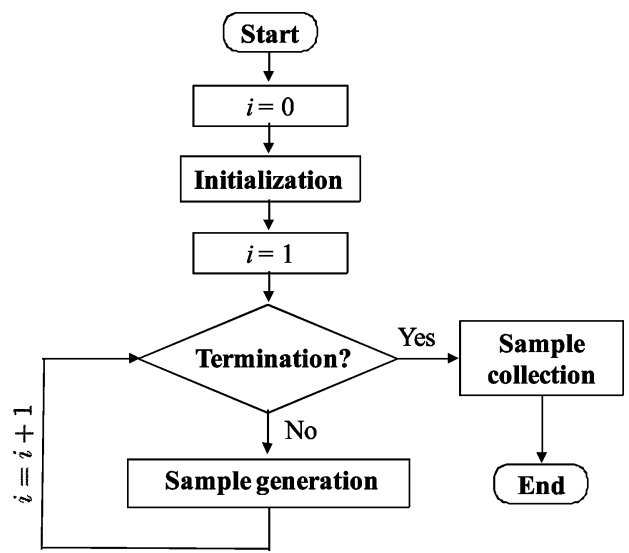

Fig. 2. Flowchart depicting the structure of Gibbs-Sampler used in the SDM/ SDMA system.

1) Initialization: The initialization block of the Gibbs-Sampler of Fig. 2 randomly generates binary signals $\boldsymbol{x}[i=0]=$ $\left[x_{1}[0] \cdots x_{M}[0]\right]^{T}$, which represent one of the $N_{b}=2^{M}$ legitimate signal sequences of (4) in our $\mathcal{M}$-QAM SDM/SDMA system.

2) Sample Generation: In the sample generation block of Fig. 2, the signals $\boldsymbol{x}[i]=\left[x_{1}[i] \cdots x_{M}[i]\right]^{T}$ generated during the $i$ th loop are calculated based on the $(i-1)$ st signals $x[i-1]$, on the a priori LLRs $\boldsymbol{L}_{1}^{p r}$, on the received signals $\boldsymbol{y}$ and on the estimated channels $\boldsymbol{H}^{\prime}$. To be more specific, the $m$ th element $x_{m}[i]$ of the signals $\boldsymbol{x}[i]$ is calculated from the conditional probability [11]

$$
\begin{aligned}
P & \left(x_{m}=+1 \mid \boldsymbol{x}_{-m}, \boldsymbol{y}, \boldsymbol{L}_{1}^{p r}\right) \\
& =\frac{1}{\left(1+\frac{P\left(x_{m}=-1, \boldsymbol{x}_{-m} \mid \boldsymbol{L}_{1}^{p r}\right) p\left(\boldsymbol{y} \mid x_{m}=-1, \boldsymbol{x}_{-m}\right)}{P\left(x_{m}=+1, \boldsymbol{x}_{-m} \mid \boldsymbol{L}_{1}^{p r}\right) p\left(\boldsymbol{y} \mid x_{m}=+1, \boldsymbol{x}_{-m}\right)}\right)}
\end{aligned}
$$

with

$$
p\left(\boldsymbol{y} \mid x_{m}= \pm 1, \boldsymbol{x}_{-m}\right)=\frac{1}{\left(\sqrt{2 \pi} \sigma_{n}^{\prime}\right)^{N}} \exp \left(-\frac{\left\|\boldsymbol{y}-\boldsymbol{H}^{\prime} \tilde{\boldsymbol{x}}\right\|^{2}}{2 \sigma_{n}^{\prime 2}}\right)
$$

where we have $\boldsymbol{x}_{-m}=\left[x_{1}[i], \ldots, x_{m-1}[i], x_{m+1}[i-\right.$ $\left.1], \ldots, x_{M}[i-1]\right]^{T}$ and $\tilde{\boldsymbol{x}}=\left[x_{1}[i], \ldots, x_{m-1}[i], x_{m}, x_{m+1}[i-\right.$ $\left.1], \ldots, x_{M}[i-1]\right]^{T}$. When a real-valued random variable $\zeta$, which is uniformly distributed between 0 and 1 , happens to be lower than the probability $P\left(x_{m}=+1 \mid x_{-m}, \boldsymbol{y}, \boldsymbol{L}_{1}^{p r}\right)$, the $m$ th element $x_{m}[i]$ is set to +1 , otherwise, to -1 . This sample generation block is activated for $N_{s}$ iterations; thus, a total of $N_{s}$ signals $\boldsymbol{x}[i]\left(i=1, \ldots, N_{s}\right)$ are generated.

3) Sample Collection: Finally, in the sample collection block, the signals generated in the last $N_{\mathrm{MC}}$ iterations are collected as the most likely detection solutions identified by the Gibbs-Sampler. Here, the first $N_{\text {burn }}=N_{s}-N_{\mathrm{MC}}$ iterations are selected as the burn-in period indicating that these initial detection candidates are typically discarded, which allows the solution to converge at the most likely values from the randomly generated initial solutions $\boldsymbol{x}[0]$.

It is clear that the $N_{\mathrm{MC}}$ number of detection candidates generated by the Gibbs-Sampler of Fig. 2 are mutually correlated since all of the signals are originated from the initial conditions $x[0]$. Therefore, $N_{\mathrm{P}}$ parallel Gibbs-Samplers may be invoked to avoid the problem of having highly correlated successive Gibbs-Sampler solutions [10]. The employment of this method results in an increased number of detection candidates $N_{\mathrm{MC}} N_{\mathrm{P}}$.

Having completed the generation of the Gibbs-Sampler's detection candidate set of $N_{\mathrm{MC}} N_{\mathrm{P}}$ signals, only $N_{b}^{\prime}<N_{b}$ number of detection candidates $\boldsymbol{x}^{\left(q^{\prime}\right)}$ are retained from the Gibbs-Sampler solution set, also ensuring that the identical detection candidates of the parallel Gibbs-Samplers are removed, which results in $N_{b} \leq N_{\mathrm{MC}} N_{\mathrm{P}}$. Note that using a single Gibbs-Sampler may generate even identical detection candidates, especially when the absolute values of the a priori LLRs and/or the SNR are sufficiently high. Then, these $N_{b}^{\prime}<N_{b}$ detection candidates are used for calculating the gradient in (8) by replacing $N_{b}$ and $\boldsymbol{x}^{(q)}$ by $N_{b}^{\prime}<N_{b}$ and $x^{(q)^{\prime}}$, respectively. Typically, $N_{b}^{\prime}$ becomes significantly lower than $N_{b}=2^{M}$, which is an explicit benefit of the rapid convergence of the Gibbs-Sampler detailed in Fig. 2.

The computational complexity of the MC-MBER detector, which is required for calculating the gradient of the BER with respect to the weights in (8), can be reduced by a factor of $N_{b} / N_{b}^{\prime}$ in comparison to that of the full-complexity MBER scheme, although the MC-MBER detector imposes the additional computation of the Gibbs-Sampler-based reduced set of $N_{b}^{\prime}<N_{b}$ signals.

\section{Performance AnAlysis}

In this section, we present our performance results characterizing the proposed MC-MBER aided system employing $N_{\mathrm{rx}}=$ 2 receive antennas at the $\mathrm{BS}$ and supporting $N_{u}=2$ users, each having $N_{\mathrm{tx}}=2$ transmit antennas and using 4-QAM transmissions, which results in the $(N \times M)=(4 \times 8)$-element equivalent channel matrix $\boldsymbol{H}^{\prime}$. For comparison, the performance of the MMSE and MBER detectors is also considered. Each user has a different random interleaver having a length of 200000 bits and employs the same half-rate recursive systematic convolutional (RSC) code having a constraint length of 5 and the octally represented generator polynomials of $(35,23)$. For each user, $N_{\mathrm{tx}}=2$ number of 4-QAM modulated symbols are transmitted over frequency flat Rayleigh fading channels, where the total bandwidth efficiency is 4 bits/s/channel use, corresponding to $4 \mathrm{bits} / \mathrm{s} / \mathrm{Hz}$ in case of zero Nyquist access bandwidth.

\section{A. BER Performance}

Fig. 3 shows the achievable BER performance of the MC-MBER detector in conjunction with both $N_{\mathrm{P}}=1$ and $N_{\mathrm{P}}=5$, as well as that of the MMSE and MBER detectors. The other parameters of the Gibbs-Sampler were set to $N_{\text {burn }}=5$ and $N_{\mathrm{MC}}=10$. Furthermore, the corresponding perfect cancellation based single-stream bound is also plotted in Fig. 3. The MC-MBER curves of both $N_{\mathrm{P}}=1$ and $N_{\mathrm{P}}=5$ exhibit good BER results, which are close to that of the full-complexity MBER detector, while MMSE detector exhibits $4 \mathrm{~dB}$ worse performance at the BER of $10^{-5}$ in this challenging rank-deficient scenario. It can be also concluded that the MC-MBER detector's performance in this simulation is essentially unaffected by the number of parallel chains $N_{\mathrm{P}}$. This is owing to the fact that the MC-MBER is capable of directly reducing the BER, as far as the $N_{b}^{\prime}$ number of detection candidates includes the transmitted signals to be detected. This is beneficial in terms of reducing the computational complexity imposed, since the total number of parallel chains is reduced. 


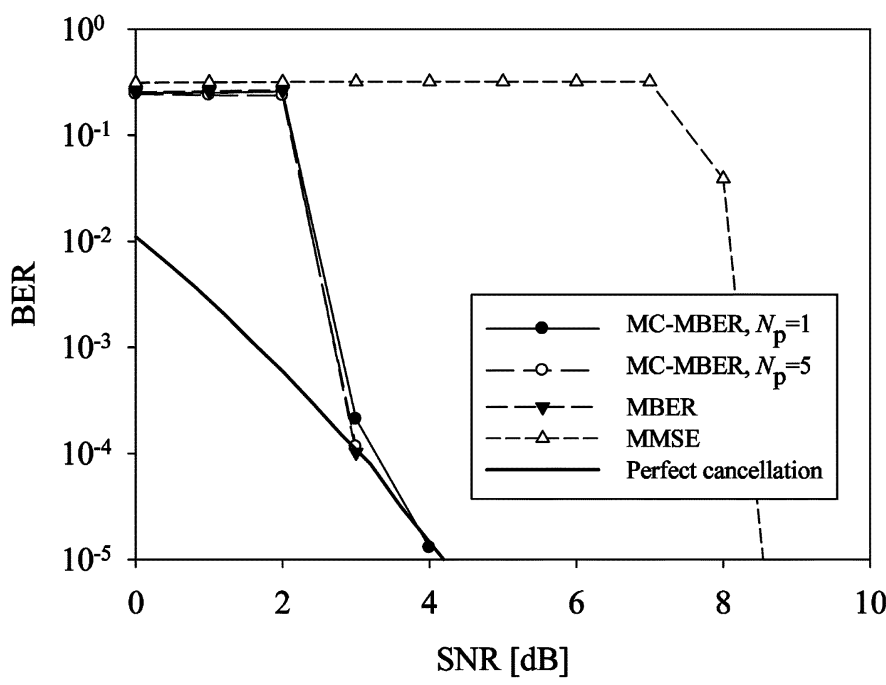

Fig. 3. BER performance of the MMSE, MBER, and MC-MBER detectors using $I=7$ iterations in a 4-QAM SDM/SDMA scenario employing $N_{u}=2$, $N_{\mathrm{tx}}=2$, and $N_{\mathrm{rx}}=2$. The MC-MBER detector invoked $N_{\mathrm{P}}=1$ or $N_{\mathrm{P}}=5$ Markov chains having a burn-in period of $N_{\mathrm{burn}}=5$ and $N_{\mathrm{MC}}=10$ signals generated in a single Gibbs-Sampler.

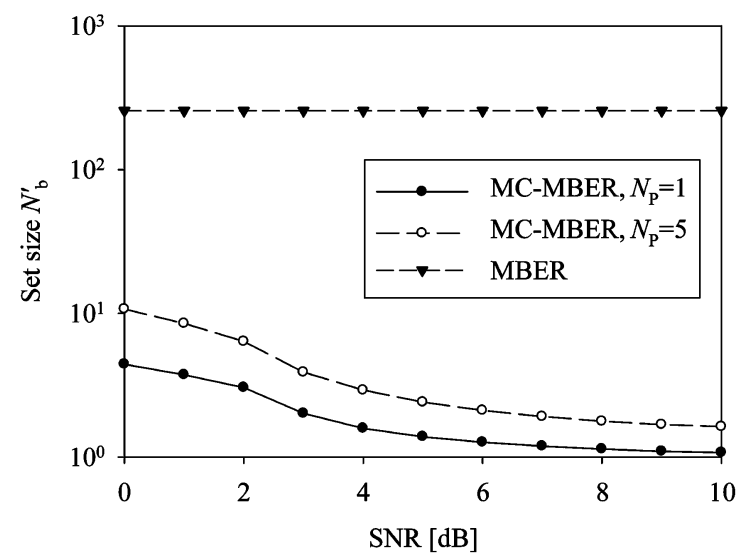

Fig. 4. Number of detection candidates $N_{b}^{\prime}$ generated by the Gibbs-Sampler and used for calculating the gradient of the MC-MBER weights in (8), where $N_{b}^{\prime}$ was averaged over all the iterations $I$ at each SNR. The value of $N_{b}=$ $2^{M}=2^{8}=256$ used for the full-complexity MBER detector is also plotted.

\section{B. Computational Complexity}

Fig. 4 shows the number of detection candidates $N_{b}^{\prime}$ used for calculating the gradient of the MC-MBER weights in (8), where the number $N_{b}^{\prime}$ was averaged over all the iterations $I$ at each SNR. The number $N_{b}=2^{M}=2^{8}=256$ used for the full-complexity MBER scheme is also plotted in Fig. 4. For the MC-MBER scheme, we had $N_{b}^{\prime}<N_{b}$. More specifically, in case of $N_{\mathrm{P}}=1$, the number of detection candidates $N_{b}^{\prime}$ tended to be unity upon increasing the SNR.

Table I shows the computational complexity required to calculate the weights $\boldsymbol{w}_{m}$ for the full-complexity MBER and for the MC-MBER detectors at the SNR $=3 \mathrm{~dB}$ in our SDM/SDMA system. The complexity was evaluated in terms of the number of real-valued operations, expressed as the sum of real-valued multiplications and real-valued additions. Furthermore, since the MC-MBER detector requires both the Gibbs-Sampler and the SCG algorithms, both their complexity is characterized as
TABLE I

COMPLEXITY AT SNR $=3 \mathrm{~dB}$ IN THE SDM/SDMA SYSTEM

\begin{tabular}{|c|c|c|c|c|}
\hline \multirow{2}{*}{ MUD } & \multicolumn{3}{|c|}{ Complexity } & \multirow{2}{*}{ BER } \\
\cline { 2 - 4 } & $\begin{array}{c}\text { Gibbs- } \\
\text { Sampler }\end{array}$ & SCG & Total & \\
\hline \hline MC-MBER, $N_{\mathrm{P}}=1$ & 68,642 & 36,932 & 105,574 & $2.1 \times 10^{-4}$ \\
\hline MC-MBER, $N_{\mathrm{P}}=5$ & 343,203 & 41,011 & 384,214 & $1.2 \times 10^{-4}$ \\
\hline MBER & - & 813,924 & 813,924 & $1.0 \times 10^{-4}$ \\
\hline
\end{tabular}

well as the total complexity in Table I. The total complexity of the MC-MBER detector using $N_{\mathrm{P}}=1$ was found to be a factor of eight lower than that of the full-complexity MBER scheme. Additionally, it is seen in both the MC-MBER detectors using both $N_{\mathrm{P}}=1$ and $N_{\mathrm{P}}=5$ that the Gibbs-Sampler constitutes the dominant factor in the total complexity in comparison to the SCG algorithm.

\section{CONCLUSION}

In this letter, we proposed a reduced-complexity Markov chain representation aided MBER detector designed for the SDM/SDMA uplink. Our simulation results revealed that the complexity of the MC-MBER MUD is a factor of eight lower than that of the MBER MUD in an 4-QAM modulated rank-deficient system having $N_{\mathrm{rx}}=2$ receive antennas and $N_{u}=2$ users, each employing $N_{\mathrm{tx}}=2$ receive antennas, while keeping the BER performance comparable to that of the MBER MUD.

It is also predicted that the complexity advantage of the MC-MBER scheme over the MBER scheme increases upon increasing $M$, since the number of detection candidates $N_{b}^{\prime}$ does not increase exponentially.

\section{REFERENCES}

[1] C. Berrou, A. Glavieux, and P. Thitimajshima, "Near shannon limit error-correcting coding and decoding: Turbo-codes," in Proc. IEEE ICC1993, Geneva, vol. 2, pp. 1064-1070.

[2] X. Wang and H. Poor, "Iterative (turbo) soft interference cancellation and decoding for coded CDMA," IEEE Trans. Commun., vol. 47, no. 7, pp. 1046-1061, Jul. 1999.

[3] M. Tüchler, A. Singer, and R. Koetter, "Minimum mean squared error equalization using a priori information," IEEE Trans. Signal Process., vol. 50, no. 3, pp. 673-683, Mar. 2002.

[4] S. Chen, A. Samingan, B. Mulgrew, and L. Hanzo, "Adaptive minimum-BER linear multiuser detection for DS-CDMA signals in multipath channels," IEEE Trans. Signal Process., vol. 49, no. 6, pp. 1240-1247, Jun. 2001.

[5] S. Chen, A. Livingstone, and L. Hanzo, "Minimum bit-error rate design for space-time equalization-based multiuser detection," IEEE Trans. Commun., vol. 54, no. 5, pp. 824-832, May 2006.

[6] S. Tan, S. Chen, and L. Hanzo, "On multi-user EXIT chart analysis aided turbo-detected MBER beamformer designs," IEEE Trans. Wireless Commun., vol. 7, no. 1, pp. 314-323, Jan. 2008.

[7] S. Tan, S. Chen, and L. Hanzo, "Iterative multiuser minimum symbol error rate beamforming aided QAM receiver," IEEE Signal Process. Lett., vol. 15, pp. 301-304, 2008.

[8] Z. Yang, B. Lu, and X. Wang, "Bayesian Monte Carlo multiuser receiver for space-time coded multicarrier CDMA systems," IEEE J. Select. Areas Commun., vol. 19, no. 8, pp. 1625-1637, Aug. 2001.

[9] R. Chen, J. Liu, and X. Wang, "Convergence analyses and comparisons of Markov chain Monte Carlo algorithms in digital communications," IEEE Trans. Signal Process., vol. 50, no. 2, pp. 255-270, Feb. 2002

[10] A. Doucet and X. Wang, "Monte Carlo methods for signal processing: A review in the statistical signal processing context," IEEE Signal Process. Mag., vol. 22, no. 6, pp. 152-170, Nov. 2005.

[11] B. Farhang-Boroujeny, H. Zhu, and Z. Shi, "Markov chain Monte Carlo algorithms for CDMA and MIMO communication systems," IEEE Trans. Signal Process., vol. 54, no. 5, pp. 1896-1909, May 2006. 